\title{
Syndrome métabolique et maladies chroniques
}

\author{
D. P. Rao, M. Sc. (1, 2); S. Dai, M.D. (2); C. Lagacé, M. Sc. (2); D. Krewski, Ph. D. (1, 3)
}

Cet article a fait l'objet d'une évaluation par les pairs.

\section{Résumé}

Introduction : Le syndrome métabolique (SMét) est un ensemble de marqueurs de risques qui semble favoriser l'apparition de maladies chroniques. Nous avons examiné le fardeau lié au SMét au Canada et son association actuelle et projetée avec les maladies chroniques.

Méthodologie : Nous avons utilisé les données de l'Enquête canadienne sur les mesures de la santé 2007-2009 pour déterminer la prévalence du SMét chez les adultes canadiens et pour examiner les associations entre divers facteurs sociodémographiques et les principales maladies chroniques. Nous avons estimé l'incidence cumulative projetée du diabète et le risque, en pourcentage, d'événements cardiovasculaires mortels en utilisant l'algorithme DPoRT (Diabetes Population Risk Tool) et l'algorithme de Framingham.

Résultats : Après ajustement en fonction de l'âge, nous avons pu déterminer que 14,9\% des adultes canadiens présentaient un SMét. Les taux étaient similaires pour les deux sexes, mais ils étaient plus élevés chez les non-Blancs et chez les personnes présentant un embonpoint ou obèses ( $p<0,001$ dans les trois cas). L'importance du SMét sur le plan de la santé publique découle du fait qu'il est associé de manière statistiquement significative avec des maladies chroniques, en particulier avec le diabète de type 2 diagnostiqué $(11,2 \%$ contre $3,4 \%)$ et non diagnostiqué $(6,0 \%$ contre $1,1 \%)$. Le taux estimé d'incidence sur 10 ans associé au diabète et le risque moyen en pourcentage d'événements cardiovasculaires mortels étaient plus élevés chez les personnes atteintes de SMét que chez celles qui ne l'étaient pas (18,0\% contre 7,1\% pour le diabète et 4,1\% contre $0,8 \%$ pour les maladies cardiovasculaires).

Conclusion : Le SMét est répandu chez les adultes canadiens, et une forte proportion de personnes souffrant d'un SMét ont également des affections chroniques, diagnostiquées ou non. Les estimations projetées du taux d'incidence de maladies chroniques associées à un SMét sont plus élevées chez les personnes aux prises avec ce syndrome. Par conséquent, le SMét pourrait être un facteur de risque pertinent dans l'apparition de maladies chroniques.

\section{Introduction}

La grande majorité des patients au sein du système de santé canadien vit avec une ou plusieurs maladies chroniques ${ }^{1}$. Les maladies cardiovasculaires, la bronchopneumopathie chronique obstructive, le cancer et le diabète sont les principales causes d'hospitalisation et de décès prématuré au Canada, avec près des trois quarts des décès attribua- bles à ces maladies ${ }^{2}$. Prises ensemble, ces maladies chroniques représentent $80 \%$ des consultations pour soins de santé primaires et plus des deux tiers des coûts associés aux soins de santé ${ }^{1,3}$. Par conséquent, en en sachant davantage sur les facteurs de risque et sur les indicateurs de maladie chronique, nous serions mieux à même de soutenir les efforts de santé publique tournés vers ce sujet croissant de préoccupation.
Le syndrome métabolique (SMét) englobe des marqueurs de risque qui augmentent la probabilité qu'une personne soit atteinte d'une maladie chronique ${ }^{4}$. Un certain nombre de maladies chroniques importantes se sont en effet avérées associées au SMét. Il s'agit notamment des maladies cardiovasculaires $(\mathrm{MCV})^{5}$, du diabète de type $2^{6}$, du cancer ${ }^{7}$ et des néphropathies chroniques ${ }^{8}$.

L'augmentation du nombre de personnes obèses et de personnes sédentaires contribue à la prévalence du SMét ${ }^{9-11}$. Si la pathogenèse du SMét peut être attribuée à l'obésité et à une prédisposition métabolique $^{12}$, certains facteurs socioéconomiques influent aussi sur la prévalence du SMét. Par exemple, les adultes canadiens ayant fait des études supérieures ont moitié moins de risques d'être atteints d'un sMét que ceux avec un diplôme d'études secondaires (rapport de cotes [RC] : 0,45, intervalle de confiance [IC] à $95 \%: 0,25$ à 0,81$)^{13}$. L'origine ethnique a aussi une incidence sur les taux de prévalence observés (RC : 0,54, IC à $95 \%$ : 0,4 à 0,73 chez les Noirs non hispaniques par rapport aux Blancs non hispaniques $)^{14}$. Un certain nombre de définitions officielles du SMét ont été adoptées par les autorités sanitaires internationales pour tenir compte de ces différences sur le plan de l'origine ethnique $^{4,15,16}$. On a également décrit le SMét comme une affection progressive, les diverses composantes du SMét ayant tendance à s'aggraver au fil du temps et contribuant collectivement à une augmentation du risque de maladie chronique ${ }^{17}$.

Hivert et collab. ${ }^{18}$ ont démontré l'utilité du SMét comme outil en santé publique. En

Rattachement des auteurs :

1. Institut de recherche sur la santé des populations, Université d'Ottawa, Ottawa (Ontario), Canada

2. Agence de la santé publique du Canada, Ottawa (Ontario), Canada

3. Risk Sciences International, Ottawa (Ontario), Canada

Correspondance : Deepa Rao, Institut de recherche sur la santé des populations, Université d’Ottawa, 1, rue Stewart, suite 300, Ottawa (Ontario) K1N 6N5; tél. : 613-897-8111; téléc. : 613-562-5380; courriel : Deepa.Rao@uottawa.ca 
utilisant des dossiers de santé électroniques pour repérer les patients atteints d'un SMét et pour surveiller l'apparition d'une $\mathrm{MCV}$ et du diabète, ils ont montré que la fréquence de ces affections chroniques et les coûts associés aux soins de santé qui en découlent étaient plus élevés chez les patients atteints d'un SMét que chez ceux qui ne l'étaient pas ${ }^{18}$. Le rôle du SMét en tant qu'indicateur de maladie chronique pourrait donc être important et se révéler bénéfique sur le plan de la santé individuelle et sur le plan des coûts et des ressources associés aux soins de santé ${ }^{18}$. Les estimations de la prévalence fondées sur des données canadiennes étant limitées, on utilise souvent des estimations internationales. Générer des données canadiennes sur le SMét et sur son association avec diverses maladies chroniques se révèle donc important.

Dans cette étude, nous visions à estimer le taux de prévalence du SMét au sein de la population canadienne d'âge adulte, à examiner le lien entre le SMét, divers facteurs de risques et les maladies chroniques et à caractériser le risque futur de maladie chronique associé au SMét à l'aide de mesures portant sur les maladies non diagnostiquées et à l'aide de projections sur 10 ans concernant le diabète et les MCV réalisées avec des outils prédictifs fiables.

\section{Méthodologie}

\section{Source des données}

Nous avons utilisé les données de l'Enquête canadienne sur les mesures de la santé (ECMS) 2007-2009 $9^{19}$. Pour cette enquête transversale, menée par Statistique Canada, on a constitué un échantillon représentatif de 5600 Canadiens de 6 à 79 ans, qui représentait environ 96,3\% de la population canadienne. On a utilisé également un centre d'examen mobile pour mesurer, entre autres, la pression artérielle et les concentrations de divers facteurs sériques chez les participants. L'information sur l'état de santé, les variables socioéconomiques et autres a été recueillie au moyen d'entrevues réalisées auprès des membres des ménages ${ }^{19}$. Statistique Canada a attribué un facteur de pondération à chaque participant afin de refléter le nombre de personnes dans la population représentées par le participant en question et en tenant également compte du taux de non-réponse et de la distribution de la population. Des renseignements plus détaillés sur l'échantillonnage et les estimations sont fournis ailleurs $^{20,21}$.

\section{Population à l'étude}

On a demandé à certains participants de l'ECMS ( $n=2$ 634) de se présenter au centre d'examen mobile à jeun pour se soumettre aux tests, et ce sont les données associées à ce sous-échantillon que nous avons utilisées ici. Le taux de réponse a été de $85,2 \%$, ce qui, combiné au taux de réponse global pour l'ensemble de l'ECMS, a donné un taux de réponse global de 46,3\% pour ce sous-échantillon de participants à jeun ${ }^{19,20,22}$. Les femmes enceintes $(n=8)$ et les moins de 20 ans ( $\mathrm{n}=933$ ) ont été exclues de l'analyse, de sorte que l'étude comptait au total 1693 participants. Statistique Canada a fourni des facteurs de pondération distincts, fondés sur le recensement de 2006, pour que les analyses menées sur cette souspopulation de participants à jeun demeurent représentatives de la population canadienne dans son ensemble. Ces facteurs de pondération tiennent compte du taux de non-réponse et de la distribution de la population au Canada. Les valeurs manquantes ont été retirées avant l'analyse.

Pour déterminer s'il y avait un biais de sélection découlant des multiples critères d'exclusion, nous avons effectué une analyse de sensibilité et comparé le statut de notre population à l'étude avec les estimations nationales. Nous avons constaté que les deux étaient similaires dans le cas de l'âge ${ }^{23}$, de la scolarité ${ }^{24}$, $\mathrm{du} \operatorname{sexe}^{25}$, de l'origine ethnique $\mathrm{e}^{26}$ et du revenu $^{27}$ (tableau 1), ce qui signifie que la population à l'étude est représentative de l'ensemble de la population canadienne.

\section{Définitions clés}

\section{Syndrome métabolique}

Nous avons utilisé la définition du SMét du National Cholesterol Education Program (rNCEP) Adult Treatment Panel III révisé, qui utilise des critères révisés pour la circonférence de la taille ${ }^{4}$. Nous avons également examiné les taux de prévalence du SMét en utilisant la définition de l'International Diabetes Federation (IDF) et la définition dite " harmonisée ${ }^{15,16}$.

\section{Maladies chroniques non diagnostiquées et diagnostiquées}

En l'absence de données longitudinales permettant de vérifier si les personnes atteintes d'un SMét développent certaines maladies chroniques au fil du temps, nous avons déterminé si les participants étaient atteints ou non d'une maladie non diagnostiquée. Nous avons considéré qu'il s'agissait d'une mesure substitut du risque futur de maladie chronique. Les participants étaient considérés comme ayant une maladie non diagnostiquée s'ils disaient ne pas être atteints de la maladie en question mais qu'ils présentaient certaines caractéristiques physiques mesurables de cette maladie.

L'hypertension diagnostiquée était fondée sur une réponse positive à la question " Faites-vous de l'hypertension (de la haute pression)? » ou par l'utilisation autodéclarée de certains médicaments (une liste peut être fournie par les auteurs sur demande). La pression systolique moyenne et la pression diastolique moyenne ont été calculées à partir de six mesures de la pression artérielle $e^{22,28,29}$. Nous avons considéré que les participants présentaient une hypertension non diagnostiquée s'ils disaient ne pas avoir reçu de diagnostic d'hypertension, mais que leur pression artérielle était supérieure à 140/90 mmHg (pour l'une ou l'autre des mesures).

Le diabète (de type 2) diagnostiqué était fondé sur une réponse positive aux questions « Êtes-vous atteint du diabète? » ou « A-t-on diagnostiqué chez vous le diabète non insulino-dépendant (Type 2)? » ou par l'utilisation autodéclarée de certains médicaments (une liste peut être fournie par les auteurs sur demande ${ }^{22}$. Comme dans le cas de la pression artérielle, nous avons considéré que les participants présentaient un diabète non diagnostiqué s'ils avaient répondu non à l'une des questions précédentes, mais que leur glycémie à jeun était supérieure ou égale à 7,0 $\mathrm{mmol} / \mathrm{L}$. Les personnes atteintes d'un diabète de type 1 n'étaient pas visées par l'analyse. 
TABLEAU 1

Caractéristiques de la population à l'étude $(\mathrm{N}=1693)$

\begin{tabular}{|c|c|c|c|}
\hline Caractéristiques & $\mathbf{N}$ & $\%$ & IC à $95 \%$ \\
\hline \multicolumn{4}{|l|}{ Sexe } \\
\hline femmes & 886 & 50,4 & 49,8 à 50,9 \\
\hline hommes & 807 & 49,6 & 49,1 à 50,2 \\
\hline \multicolumn{4}{|l|}{ Âge (ans) } \\
\hline 20 à 39 & 536 & 37,8 & 37,1 à 38,4 \\
\hline 40 à 59 & 603 & 41,3 & 40,8 à 41,8 \\
\hline 60 à 80 & 554 & 20,9 & 20,6 à 21,2 \\
\hline Âge moyen (ET) (années) & $45,3(0,2)$ & & \\
\hline \multicolumn{4}{|l|}{ Appartenance culturelle/origine ethnique } \\
\hline Blancs & 1441 & 84,3 & 74,2 à 94,4 \\
\hline non-Blancs & 205 & $15,7^{\mathrm{E}}$ & 5,6 à 25,8 \\
\hline \multicolumn{4}{|l|}{ Revenu total du ménage (\$) } \\
\hline 29999 et moins & 290 & 14,6 & 11,6 à 17,7 \\
\hline 30000 à 49999 & 324 & 18,4 & 16,3 à 20,5 \\
\hline 50000 à 79999 & 400 & 26,4 & 22,5 à 30,3 \\
\hline 80000 et plus & 583 & 40,6 & 33,6 à 42,9 \\
\hline \multicolumn{4}{|l|}{ Niveau de scolarité le plus élevé } \\
\hline pas de diplôme d'études secondaires & 206 & 11,4 & 7,6 à 15,2 \\
\hline diplôme d'études secondaires & 289 & 18,8 & 13,1 à 24,5 \\
\hline études postsecondaires partielles/diplôme d'études postsecondaires & 1178 & 69,8 & 61,5 à 78,2 \\
\hline \multicolumn{4}{|l|}{ Statut tabagique } \\
\hline n'ayant jamais fumé & 810 & 45,7 & 41,8 à 49,5 \\
\hline ex-fumeur & 553 & 31,2 & 27,9 à 34,5 \\
\hline fumeur (quotidiennement ou à l'occasion) & 325 & 23,1 & 20,6 à 25,6 \\
\hline \multicolumn{4}{|l|}{ Activité physique durant les loisirs } \\
\hline actif ou modérément actif & 800 & 44,3 & 37,2 à 51,5 \\
\hline inactif & 893 & 55,7 & 48,5 à 62,8 \\
\hline \multicolumn{4}{|l|}{ IMC $\left(\mathrm{kg} / \mathrm{m}^{2}\right)$} \\
\hline moins de 25 & 676 & 43,5 & 37,8 à 49,2 \\
\hline 25 à 29 & 638 & 37,8 & 33,8 à 41,8 \\
\hline 30 et plus & 351 & 18,7 & 15,6 à 21,2 \\
\hline
\end{tabular}

Source : Enquête canadienne sur les mesures de la santé 2007-2009, ensemble de données cliniques.

Abréviations : ECMS, Enquête canadienne sur les mesures de la santé; ET, erreur type; IC, intervalle de confiance; IMC, indice de masse corporelle.

Remarques : Les données manquantes (sans objet, non mentionné, inconnu) ne sont pas incluses dans le calcul des proportions. Les pourcentages ont été pondérés selon la pondération utilisée dans l'ECMS.

E Interpréter avec prudence (coefficient de variation compris entre 16,6 \% et 33,3\%).

La néphropathie chronique diagnostiquée était fondée sur une réponse positive à la question "Souffrez-vous d'une dysfonction ou d'une maladie rénale? $»^{22}$. Nous avons considéré que les participants présentaient une néphropathie chronique non diagnostiquée s'ils répondaient à cette question par la négative, mais que le débit de filtration glomérulaire mesuré était faible $(60 \mathrm{~mL} / \mathrm{min}$ ou moins selon l'équation de Modification of Diet and Renal
Disease Study ${ }^{30}$ ) ou que le rapport microalbumine/créatinine était élevé (supérieur à 2,65 mg/mmol).

La dyslipidémie diagnostiquée était fondée sur une réponse positive à la question « Un professionnel de la santé vous a-t-il déjà dit que votre taux de cholestérol sanguin était élevé? ${ }^{22}$. Nous avons considéré que les participants présentaient une dyslipidémie non diagnostiquée s'ils répondaient à la question précédente par la négative mais que soit ils remplissaient à la fois le critère relatif au rapport cholestérol total/ cholestérol à lipoprotéines de haute densité (cholestérol-HDL) (5,5 ou plus chez les hommes; 4,5 ou plus chez les femmes) et celui relatif aux lipoprotéines de basse densité (LDL) (3,5 mmol/L ou plus), soit ils prenaient certains médicaments (liste fournie par les auteurs sur demande).

\section{Variables}

Les analyses sont présentées en fonction du sexe, de l'âge (au moment de la consultation en centre d'examen), de la scolarité, de l'origine ethnique (groupe racial ou culturel autodéclaré, populations autochtones non incluses) et du revenu du ménage. Les facteurs relatifs au style de vie sont l'indice de masse corporelle (IMC) mesuré et les déclarations des participants relativement à leur activité physique durant leurs loisirs et à leur statut tabagique ${ }^{19}$.

\section{Analyse statistique}

Nous avons effectué des analyses multivariées à l'aide du logiciel statistique SAS Enterprise Guide 4.1 (Cary, Caroline du Nord, États-Unis) ${ }^{31}$. Les estimations nationales ont été établies à l'aide de la pondération utilisée pour l'ECMS pour le sous-échantillon de population à jeun et nous les avons ajustées selon l'âge à l'aide des données du recensement canadien. Nous avons estimé la variance à l'aide du logiciel Bootvar de Statistique Canada et en suivant les lignes directrices de Statistique Canada en matière de déclaration. Nous avons utilisé l'estimateur de Horvitz-Thompson pour analyser la signification statistique suivant une distribution de Student à 11 degrés de liberté.

Nous avons examiné les estimations de la prévalence à l'aide de la procédure de fréquence du logiciel SAS Enterprise Guide 4.1 et les avons ajustées (voir la section Résultats pour leur description). Les RC estimatifs, lorsqu'ils sont mentionnés, ont été calculés à l'aide de modèles de régression logistique et ajustés selon l'âge et le sexe. Les projections de l'incidence cumulative sur 10 ans pour le diabète de type 2 ont été estimées à l'aide de l'outil DPoRT (Diabetes Population Risk Tool) ${ }^{32}$. Cet 
outil prédictif, initialement élaboré en utilisant l'Enquête nationale sur la santé de la population, utilise des données d'enquête courantes (par exemple les estimations autodéclarées relatives aux comportements en matière de santé et les facteurs sociodémographiques) pour prévoir le risque pour un sujet de recevoir un diagnostic médical de diabète. Nous avons utilisé les modèles de survie de Weibull en fonction du sexe pour créer un DPoRT pour les personnes non atteintes de diabète sucré, de plus de 20 ans et qui ne sont pas enceintes. Les variables prédictives utilisées dans le modèle étaient l'âge, le sexe, l'origine ethnique autodéclarée, l'IMC autodéclarée, le statut d'immigrant (pour les femmes), la scolarité, le statut tabagique et les antécédents d'hypertension artérielle et de cardiopathie, et toutes étaient disponibles pour notre analyse ${ }^{32}$.

Nous avons utilisé le calculateur de risque sur 10 ans basé sur les lipides de Framingham pour estimer le risque d'événement cardiovasculaire général mortel, défini comme une mort d'origine coronarienne, un infarctus du myocarde, une insuffisance coronarienne, un angor, un accident vasculaire cérébral ischémique, un accident vasculaire cérébral hémorragique, un accident ischémique transitoire, une artériopathie périphérique ou une insuffisance cardiaque. Cet outil prédictif a été créé à l’origine avec les données des études Framingham Heart Study et Framingham Offspring Study. Nous avons utilisé des régressions de risques proportionnels de Cox en fonction du sexe pour relier les divers facteurs de risque et l'incidence d'événements cardiovasculaires généraux mortels. Les fonctions du risque mathématique de $\mathrm{MCV}$ issues de ce processus ont ensuite été intégrées à l'outil de risque de Framingham. Les résultats sont présentés selon un niveau de risque élevé (20\% et plus) ou modéré à élevé (10\% et plus). Le sous-ensemble de la population utilisé pour les projections de MCV a été réduit aux personnes de 30 à 74 ans sans antécédent d'événement cardiovasculaire $^{33}$.

\section{Approbation éthique}

Nous avons obtenu l'approbation du Comité d'éthique de la recherche de l'Hôpital d'Ottawa (protocole n²012076701H) avant de débuter notre étude.

\section{Résultats}

La majorité des participants à l'enquête étaient blancs, physiquement inactifs et fumeurs ou ex-fumeurs. Dans la plupart des cas, ils avaient effectué un certain nombre d'années d'études postsecondaires, et le revenu annuel de leur ménage était supérieur à 50000 \$. L'âge moyen de la population à l'étude était de 45 ans, et la répartition par sexe était égale (tableau 1).

Nous avons considéré que les participants présentaient un SMét lorsqu'ils répondaient à au moins trois critères du rNCEP, ce qui s'est traduit par une prévalence brute de $15,5 \%$ et une prévalence ajustée selon l'âge de 14,9\%. Au sein de la population générale, $34,9 \%$ des personnes ne présentaient aucun marqueur de risque du SMét, 29,5 \% des personnes en présentaient un et 20,2 \% des personnes en présentaient deux. Les marqueurs de risque du SMét le plus souvent observés chez les personnes qui en étaient atteintes étaient la circonférence de la taille $(89,2 \%)$, une hypertriglycéridémie $(82,3 \%)$, un faible taux de cholestérol-HDL (75,4 \%), une glycémie à jeun élevée $(53,3 \%)$ et une pression systolique ou diastolique élevée (40,3\%) (figure 1).

Les estimations déterminées à l'aide du rNCEP ont été comparées aux estimations de prévalence fondées sur la définition de l'IDF et sur celles de la définition dite harmonisée, et dans les deux cas elles étaient significativement plus élevées (pour la prévalence brute, IDF : 23,1 \%

FIGURE 1

Prévalence des divers marqueurs de risque du syndrome métabolique chez les personnes qui en sont atteintes, ECMS 2007-2009

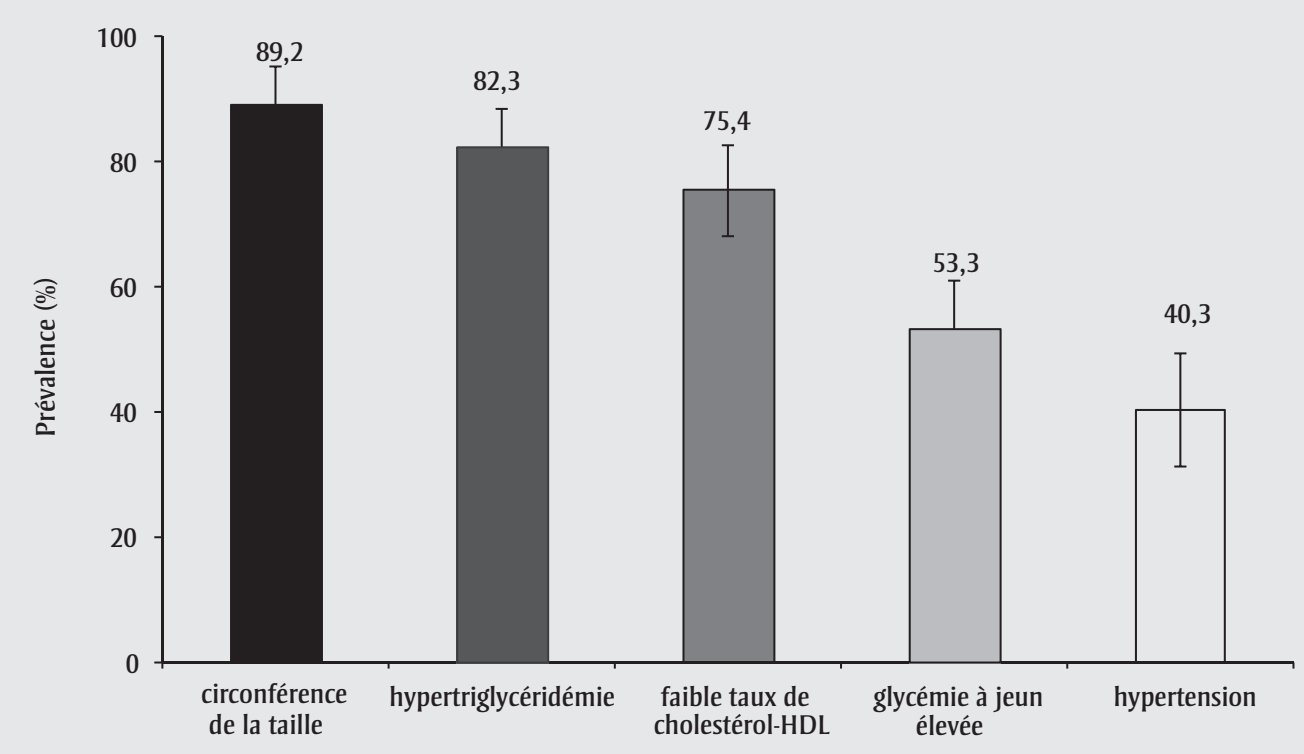

Abréviations : ECMS, Enquête canadienne sur les mesures de la santé; HDL, lipoprotéines de haute densité. 
et harmonisée : 19,6\%; pour la prévalence ajustée selon l'âge, IDF :22,3 \% et harmonisée : 19,1 \%) (tableau 2).

La prévalence du SMét variait en fonction du groupe d'âge, mais la différence entre les sexes à l'intérieur de chaque groupe d'âge n'était pas statistiquement significative (figure 2). Il y avait également une variation selon le statut tabagique, avec dans ce cas des différences entre les sexes (tableau 2). Par ailleurs, l'origine ethnique avait un effet statistiquement significatif sur les taux de prévalence, ceux-ci étant plus élevés chez les non-Blancs que chez les Blancs. Tant chez les hommes que chez les femmes, il y avait une association statistiquement significative entre un IMC élevé ou l'inactivité physique et une prévalence accrue de SMét.

Les cotes associées au SMét variaient selon les caractéristiques des participants, avec une association statistiquement significative avec le fait de ne pas être blanc et d'être plus âgé (tableau 2). Il y avait également des associations statistiquement significatives en lien avec d'autres caractéristiques, mais seulement pour l'un des sexes, par exemple des rapports de cotes relatifs au SMét statistiquement significatifs en lien avec le fait d'être fumeuse.

Nous avons examiné la prévalence de diverses maladies chroniques au sein de trois groupes : l'ensemble de la population, les personnes obèses (IMC supérieur ou égal à $30 \mathrm{~kg} / \mathrm{m}^{2}$ ) et les personnes atteintes de SMét. Les cas de maladie non diagnostiquée étaient plus fréquents chez les personnes atteintes de SMét que chez les personnes obèses ou dans l'ensemble de la population pour toutes les maladies examinées, le cas le plus flagrant étant celui de la dyslipidémie $(28,3 \%$, contre respectivement 18,5\% et $10,0 \%$ ) (tableau 3 ). Il est à noter que le taux de diabète non diagnostiqué était plus de cinq fois plus élevé chez les personnes atteintes de SMét que dans l'ensemble de la population $(6,0 \%$ contre $1,1 \%, p=0,009$; à interpréter avec prudence).

Nous avons estimé le fardeau futur associé au diabète de type 2 et aux MCV pouvant
TABLEAU 2

Prévalence et rapports de cote du syndrome métabolique selon les caractéristiques de la population, ECMS 2007-2009

\begin{tabular}{|c|c|c|c|}
\hline \multirow[t]{2}{*}{ Définitions } & \multicolumn{3}{|c|}{ Prévalence } \\
\hline & $\%$ & IC à 95 \% & valeur $p$ \\
\hline \multicolumn{4}{|l|}{ rNCEP ATP III } \\
\hline prévalence brute & 15,5 & 12,0 à 19,0 & - \\
\hline prévalence ajustée & 14,9 & 13,3 à 16,6 & \\
\hline \multicolumn{4}{|l|}{ IDF } \\
\hline prévalence brute & 23,1 & 20,4 à 25,8 & $<0,001$ \\
\hline prévalence ajustée & 22,3 & 20,4 à 24,3 & \\
\hline \multicolumn{4}{|l|}{ Harmonisée } \\
\hline prévalence brute & 19,6 & 15,9 à 23,2 & $<0,001$ \\
\hline prévalence ajustée & 19,1 & 17,3 à 20,9 & \\
\hline
\end{tabular}

\section{Caractéristiques}

Ensemble de la population

$\operatorname{Sexe}^{\mathrm{a}, \mathrm{b}}$

hommes (réf.)

femmes

Origine ethnique $\mathrm{e}^{\mathrm{a}, \mathrm{b}, \mathrm{c}}$

Blancs (réf.)

Non-Blancs

$14,5 \quad 10,4$ à 18,6

$16,5 \quad 12,6$ à 20,3

-

Rapports de cotes

RC

IC à $95 \%$

Hommes

Âge ${ }^{b}$

20 à 39 (réf.)

40 à 59

60 à 80

15,5
$16,6^{\mathrm{E}}$

12,1 à 18,8

1,12

0,87 à 1,42

5,4 à 27,7

$<0,001$

2,66

1,29 à 5,45

Statut tabagique $\mathrm{a}^{\mathrm{a}, \mathrm{b}}$

fumeur

ex-fumeur

n'ayant jamais fumé (réf.)

$\mathrm{APDL}^{\mathrm{a}, \mathrm{b}}$

actif (réf.)

inactif

IMC $\left(\mathrm{kg} / \mathrm{m}^{2}\right)^{\mathrm{a}}$

moins de 25 (réf.)

25 à 29

30 et plus

$8,0^{\mathrm{E}}$

4,4 à 11,5

$14,5^{\mathrm{E}}$

6,7 à 22,4

$-$

26,9

21,3 à 32,5

$\quad 1,48$

0,67 à 3,26

$6,6^{\mathrm{E}} \quad 2,0$ aे 11,1

$0,012 \quad 3,33$

2,07 à 5,34

6,6

2,0 à 11,1

0,01

0,65

0,23 à 1,86

$24,1 \quad 15,5$ à 32,7

0,12

1,54

0,66 à 3,61

$11,4^{\mathrm{E}} \quad 5,6$ à 17,3

-

Femmes

Âge $\mathrm{e}^{\mathrm{b}}$

20 à 39 (réf.)

40 à 59

60 à 80

\section{2}

$16,9^{\mathrm{E}}$

8,5 à 15,6

-

10,0 à 23,9

0,001

1

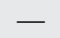

Statut tabagique $\mathrm{a}^{\mathrm{a} b}$

fumeuse

ex-fumeuse

-

15,8

38,6

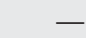

10,4 à 21,2

-
$<0,001$
$<0,001$

25,5 à $51,8<0,001$

n'ayant jamais fumé (réf.)
-

18,7

31,5

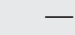

-

-

0,003

3,67

1,20 à 11,17

24,3 à 38,6

$<0,001$

7,43

$21,1 \quad 13,6$ à 28,5 $\quad 0,71 \quad 3,15 \quad 1,63$ à 6,07

$21,3^{\mathrm{E}} \quad 10,8$ à $31,8 \quad 0,38 \quad 2,06 \quad 0,93$ à 4,59

$11,0 \quad 8,7$ à $13,3 \quad-\quad 1$
2,62 à 21,05 
TABLEAU 2 (Suite)

Prévalence et rapports de cote du syndrome métabolique selon les caractéristiques de la population, ECMS 2007-2009

\begin{tabular}{|c|c|c|c|c|c|}
\hline \multirow[t]{2}{*}{ Caractéristiques } & \multicolumn{3}{|c|}{ Prévalence } & \multicolumn{2}{|c|}{ Rapports de cotes } \\
\hline & $\%$ & IC à $95 \%$ & valeur $p$ & RC & IC à $95 \%$ \\
\hline \multicolumn{6}{|l|}{$\mathrm{APDL}^{\mathrm{a}, \mathrm{b}}$} \\
\hline actif (réf.) & $10,5^{\mathrm{E}}$ & 6,6 à 14,5 & - & 1 & - \\
\hline inactif & 20,2 & 15,4 à 25,0 & $<0,001$ & 1,76 & 1,13 à 2,73 \\
\hline \multicolumn{6}{|l|}{ IMC $\left(\mathrm{kg}^{\prime} / \mathrm{m}^{2}\right)^{\mathrm{a}}$} \\
\hline moins de 25 (réf.) & $-^{\mathrm{F}}$ & - & - & 1 & - \\
\hline 25 à 29 & 22,9 & 16,1 à 30,0 & $<0,001$ & $-^{\mathrm{F}}$ & - \\
\hline 30 et plus & 43,2 & 34,2 à 52,2 & $<0,001$ & $-^{\mathrm{F}}$ & - \\
\hline
\end{tabular}

Source : Enquête canadienne sur les mesures de la santé 2007-2009, ensemble de données cliniques.

Abréviations : APDL, activité physique durant les loisirs; ECMS, Enquête canadienne sur les mesures de la santé; IC, intervalle de confiance; IDF, International Diabetes Federation; IMC, indice de masse corporelle; RC, rapport de cotes; Réf., référence; rNCEP ATP, revised National Cholesterol Education Program Adult Treatment (Panel III).

Remarque : L'estimation de la prévalence ajustée a fait l'objet d'un ajustement en fonction de l'âge selon les données du recensement canadien.

a Rapport de cotes ajusté selon l'âge.

b Rapport de cotes ajusté selon l'IMC.

c Rapport de cotes ajusté selon le sexe.

${ }^{\mathrm{E}}$ Interpréter avec prudence (coefficient de variation compris entre 16,6 \% et 33,3\%).

F Non déclarable (coefficient de variation supérieur à 33,3\%). être attribué au SMét à l'aide d'algorithmes. Le risque moyen de diabète sur 10 ans pour les personnes atteintes de SMét par rapport à celles qui ne l'étaient pas était de $18,0 \%$ (IC à $95 \%: 15,3$ à 20,7) contre 7,1\% (IC à $95 \%: 6,2$ à 8,1). Par conséquent, on peut s'attendre à ce que le pourcentage d'adultes canadiens atteints de diabète entre 2007 et 2017 soit d'environ $8,7 \%$ (IC à $95 \%: 7,5$ à 9,9) (figure 3). De même, le risque moyen d'événement cardiovasculaire mortel était de 4,1\% (IC à $95 \%: 2,3$ à 6,0; à interpréter avec prudence) contre $0,8 \%$ (IC à $95 \%$ : 0,6 à $1,0)$. Le risque de MCV sur 10 ans est considéré comme élevé s'il est supérieur ou égal à $20 \%$, et comme modéré à élevé s'il est compris entre $10 \%$ et $20 \%$. La proportion d'adultes canadiens atteints de SMét et présentant un risque élevé d'événement cardiovasculaire était de 6,81\% (IC à $95 \%: 3,2$ à 10,4, $p=0,004$ par rapport aux personnes sans SMét; à interpréter avec prudence) et celle présentant un risque modéré à élevé était de 8,9\% (IC à $95 \%$ : 4,3 à 13,6; à interpréter avec prudence), contre $2,0 \%$ (IC à $95 \%$ : 1,3 à 2,7 , $p=0,008$ ) pour les personnes sans SMét.

FIGURE 2

Prévalence du syndrome métabolique selon le sexe et le groupe d'âge, ECMS 2007-2009

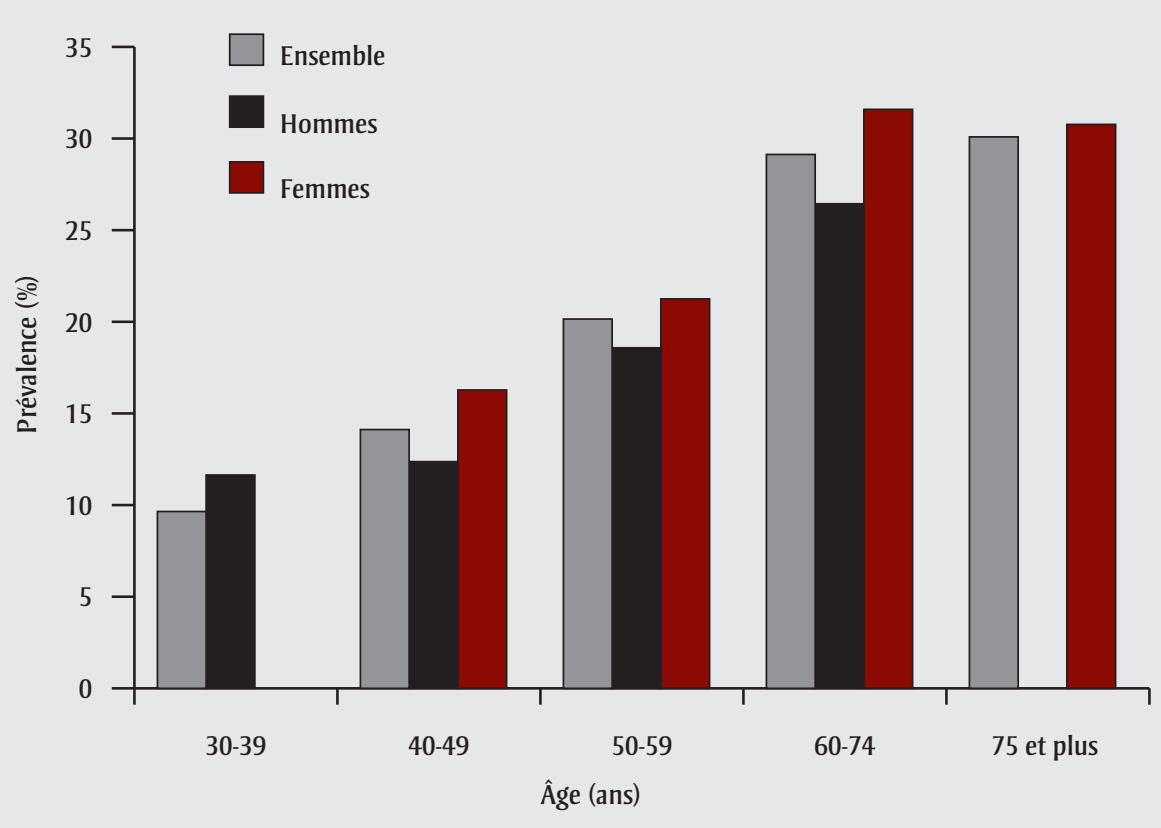

Source : Enquête canadienne sur les mesures de la santé 2007-2009, ensemble de données cliniques.

Abréviation : ECMS, Enquête canadienne sur les mesures de la santé.

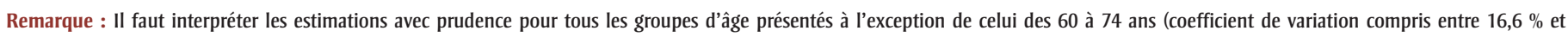
$33,3 \%$ ). Les estimations non déclarables (coefficient de variation supérieur à 33,3\%) n’ont pas été représentées. 
TABLEAU 3

Prévalence des maladies chroniques, diagnostiquées ou non, au sein de l'ensemble de la population et chez les personnes atteintes d'obésité ou de syndrome métabolique, ECMS 2007-2009

\begin{tabular}{|c|c|c|c|c|c|c|c|c|c|}
\hline & \multicolumn{2}{|c|}{$\begin{array}{l}\text { Ensemble de la } \\
\text { population }\end{array}$} & \multicolumn{3}{|c|}{ Personnes atteintes d'obésité } & \multicolumn{3}{|c|}{$\begin{array}{l}\text { Personnes atteintes de syndrome } \\
\text { métabolique }\end{array}$} & valeur $p^{b}$ \\
\hline \multicolumn{10}{|l|}{ Hypertension } \\
\hline diagnostiquée & 17,2 & 14,2 à 20,1 & 33,6 & 25,2 à 41,9 & 0,001 & 36,1 & 29,0 à 43,1 & $<0,001$ & 0,61 \\
\hline non diagnostiquée & $0,7^{\mathrm{E}}$ & 0,2 à 1,1 & $-^{\mathrm{F}}$ & - & - & $-^{\mathrm{F}}$ & - & - & - \\
\hline \multicolumn{10}{|l|}{ Diabète } \\
\hline non diagnostiqué & $1,1^{\mathrm{E}}$ & 0,6 à 1,7 & $4,4^{\mathrm{E}}$ & 1,5 à 7,2 & 0,02 & $6,0^{\mathrm{E}}$ & 2,2 à 9,8 & 0,009 & 0,27 \\
\hline \multicolumn{10}{|c|}{ Néphropathie chronique } \\
\hline diagnostiquée & 1,9 & 1,4 à 2,4 & $-^{\mathrm{F}}$ & - & - & $4,0^{\mathrm{E}}$ & 1,2 à 6,8 & 0,13 & - \\
\hline non diagnostiquée & 10,0 & 8,1 à 11,9 & $15,2^{\mathrm{E}}$ & 9,0 à 21,5 & 0,11 & 22,2 & 14,9 à 29,5 & 0,002 & 0,10 \\
\hline \multicolumn{10}{|l|}{ Dyslipidémie } \\
\hline
\end{tabular}

Source : Enquête canadienne sur les mesures de la santé 2007-2009, ensemble de données cliniques.

Abréviations : ECMS, Enquête canadienne sur les mesures de la santé; IC, intervalle de confiance.

a Ces valeurs $p$ représentent le degré de signification statistique de la différence entre les sous-groupes de la population et la population générale.

b Cette valeur $p$ représente le degré de signification statistique de la différence entre les sous-groupes de la population.

E Interpréter avec prudence (coefficient de variation : 16,6 \% à 33,3\%).

F Non déclarable (coefficient de variation : > 33,3\%).

\section{Analyse}

\section{Prévalence du syndrome métabolique}

Si l'on compare la prévalence du SMét à l'aide de la définition du rNCEP, le taux de prévalence ajusté selon l'âge au Canada est plus de deux fois moins élevé que le taux déclaré aux États-Unis (14,9\% contre $34,4 \%)^{14}$, mais similaire aux données publiées antérieurement pour la population canadienne $e^{34}$. Si l'on utilise les nouvelles définitions suggérées par l'IDF (qui tiennent compte des différences entre les groupes ethniques quant à la circonférence de la taille) ou la définition harmonisée, le taux de prévalence du SMét ajusté selon l'âge au Canada est plus élevé que le taux calculé à l'aide des définitions du rNCEP (respectivement $22,3 \%$ et $19,1 \%$ ), ce qui indique que le choix de la définition du SMét n'est pas sans conséquence.

Nous avons choisi d'utiliser la définition du rNCEP dans notre étude pour faciliter les comparaisons avec les données épidémiologiques publiées antérieurement ${ }^{14}$. Cette définition était raisonnablement pré- cise pour ce qui concerne la représentation de la composition ethnique de la population à l'étude (84\% de Blancs, tableau 1). Bien que les limites relatives à la taille de l'échantillon ne nous aient pas permis d'explorer les variations du taux de prévalence du SMét en fonction de l'origine ethnique autodéclarée, il semblerait que davantage de personnes soient considérées comme ayant un SMét lorsqu'on utilise cette information et la définition du SMét donnée par l'IDF ${ }^{15}$.

\section{Facteurs de risque et syndrome métabolique}

Selon nos observations, la prévalence du SMét au Canada est associée à l'âge, à l'origine ethnique, à l'IMC et à l'activité physique durant les loisirs. Un âge avancé était statistiquement associé au SMét, mais les profils de prévalence différaient en fonction de l'âge et du sexe. La prévalence était plus élevée chez les hommes de 30 à 39 ans. Ensuite, la prévalence du SMét augmentait de façon constante chez les femmes, pour dépasser celle observée chez les hommes entre 40 et 60 à 74 ans, puis se stabilisait. Chez les hommes, l'augmenta- tion constante de la prévalence semblait se produire après 40 ans. Selon Tjepkema ${ }^{35}$, cette transition est le reflet de l'augmentation marquée du taux d'obésité observée chez les hommes après 45 ans. Dans la même étude, Tjepkema ${ }^{35}$ a également montré que les taux d'obésité augmentaient de façon constante chez les femmes jusqu'à 65 ans. Les changements relatifs à la prévalence que nous avons observés concordent avec les taux accrus de SMét observés chez les femmes en périménopause et les femmes postménopausées ${ }^{36}$.

Les cotes relatives au SMét étaient significativement plus élevées chez les nonBlancs, et nous avons constaté que le risque de SMét était plus élevé chez les Canadiens non blancs que chez les Américains d'origine mexicaine et les Blancs non hispaniques aux États-Unis ${ }^{14}$. Outre les Canadiens d'origines hispanique et africaine, notre analyse incluait des populations d'autres origines, notamment philippine, chinoise, sud-asiatique et arabe, et il est possible que l'inclusion de ces groupes additionnels soit responsable de la différence entre les deux études observée quant aux cotes relatives au 
FIGURE 3

Projection sur 10 ans de l'incidence cumulative de diabète et du risque moyen d'événement cardiovasculaire mortel, en pourcentage, chez les personnes atteintes ou non de SMét, ECMS 2007-2009

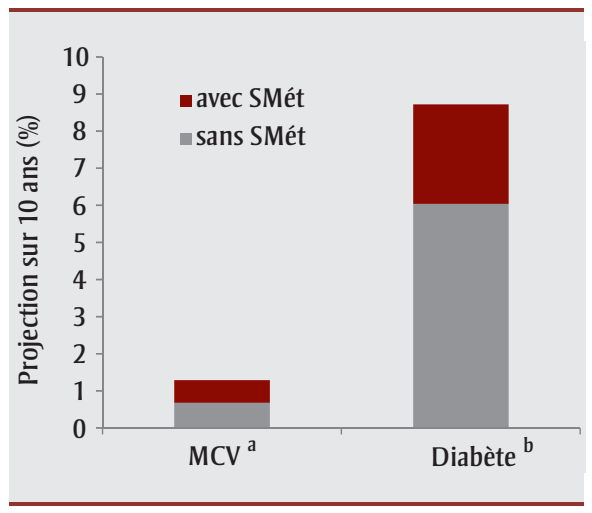

Source : Enquête canadienne sur les mesures de la santé 2007-2009, ensemble de données cliniques.

Abréviations : ECMS, Enquête canadienne sur les mesures de la santé; IC, intervalle de confiance; MCV, maladies cardiovasculaires; SMét, syndrome métabolique.

Remarques : L'incidence cumulative de diabète a été estimée à l'aide de l'outil DPoRT (Diabetes Population Risk Tool) ${ }^{32}$.

Le risque moyen d'événement cardiovasculaire mortel a été calculé à l'aide du calculateur de risque sur 10 ans basé sur les lipides selon la méthode de Framingham ${ }^{33}$. Le sousensemble de population utilisé pour les projections relatives aux MCV a été restreint aux adultes de 30 à 74 ans sans antécédent d'événement cardiovasculaire.

Étant donné la prévalence du SMét observée dans la population canadienne adulte $(85,1 \%$ sans SMét contre $14,9 \%$ avec SMét), le risque d'événement cardiovasculaire mortel projeté sur 10 ans serait de 1,29\% et l'incidence cumulative du diabète projetée sur 10 ans serait de 8,7\% (IC à $95 \%$ : 7,5 à 9,9).

${ }^{a}$ Les projections étaient, pour les MCV, de 4,1 \% avec SMét (IC à $95 \%: 2,3$ à 6,$0 ; p<0,01$ ) et de $0,8 \%$ sans SMét (IC à $95 \%: 0,6$ à 1,$0 ; p<0,01)$.

${ }^{\mathrm{b}}$ Les projections étaient, pour le diabète, de 18,0 \% avec SMét (IC à $95 \%: 15,3$ à 20,7; $p<0,01$ ) et de $7,1 \%$ sans SMét (IC à $95 \%: 6,2$ à 8,$1 ; p<0,01)$.

SMét selon l'origine ethnique. Des observations antérieures réalisées à l'aide de la définition du rNCEP montraient également que les taux de prévalence étaient plus élevés chez certains groupes ethniques inclus dans notre étude que dans la population générale utilisée ici ${ }^{37,38}$.

Selon nos résultats, les cotes relatives au SMét sont moins élevées chez les personnes actives que chez les personnes inactives, mais cette diminution du risque n'est statistiquement significative que chez les femmes. Notre analyse révèle clairement que les cas d'embonpoint et d'obésité sont fréquents chez les adultes, avec une prévalence atteignant presque $57 \%$. Il s'agit d'un fait inquiétant étant donné l'étroite association entre l'obésité et le SMét et entre l'obésité et le prédiabète $^{39}$.

Le SMét est en effet fréquemment associé au prédiabète, état qui se caractérise par une glycémie élevée et une inflammation systémique. Il est également associé à certaines caractéristiques comme un état prothrombotique ou une dyslipidémie, ce qui pourrait expliquer les liens observés avec le risque cardiovasculaire ${ }^{40}$. Le risque accru de diabète de type 2 et d'événement cardiovasculaire mortel observé chez les personnes atteintes d'un SMét n'est donc pas surprenant, plusieurs travaux de recherche montrant l'existence de telles associations ${ }^{5,41}$. La proportion de personnes considérées comme à risque d'être atteintes de diabète au cours des 10 prochaines années par rapport aux personnes sans SMét indique que le SMét pourrait servir d'indicateur de maladie chronique. Ces observations sont corroborées par une étude menée en 2010, dans laquelle on estimait à 8,9\% le risque de diabète chez les Canadiens ${ }^{42}$. Lorsqu'on tient compte des projections relatives aux $\mathrm{MCV}$, qui offrent une estimation du risque d'événement mortel, il est clair qu'il y a des raisons d'être préoccupé.

Il faut être conscient qu'un chevauchement est possible entre la définition des facteurs de risque de maladie chronique et la définition du SMét. Dans le cas de la dyslipidémie, ce chevauchement peut être à l'origine d'une augmentation des cas de lipidémie anormale chez les personnes atteintes d'un SMét. Le marqueur de risque correspondant à un faible taux de cholestérol-HDL était présent chez $75 \%$ de la population atteinte d'un SMét, mais il convient de noter que notre définition de la dyslipidémie était fondée sur un rapport élevé cholestérol total sur cholestérol-HDL associé à des niveaux élevés de LDL. Par ailleurs, la définition du SMét est basée sur la circonférence de la taille et non sur l'IMC, ce qui fait que ces deux populations sont distinctes mais potentiellement reliées.

\section{Répercussions du syndrome métabolique sur la santé publique}

Indépendamment de la race ou de l'origine ethnique, de l'âge, du sexe et de l'état de santé, les données montrent que le risque d'être atteint de certaines maladies chroniques augmente avec chaque marqueur de risque de SMét additionnel ${ }^{41}$. Selon Reaven $^{43}$, même si une personne ne présente pas le nombre de marqueurs de risque requis ( 3 ou plus) pour recevoir un diagnostic de SMét, le risque de maladie future est toujours présent et il faut en tenir compte. Nous avons observé que $50 \%$ de la population à l'étude présentait un ou deux marqueurs de risque de SMét, ce qui ne constitue aucunement une faible proportion.

Nous avons comparé le SMét avec un facteur de risque de maladie chronique bien connue : l'obésité. Selon nos observations, la prévalence de maladies chroniques était plus élevée chez les personnes atteintes de SMét que chez celles qui étaient obèses (tableau 3), mais les différences n'étaient pas statistiquement significatives. Dans une étude antérieure, on a décrit le SMét comme un meilleur indicateur de maladie future que l'obésité seule ${ }^{44}$. L'association étroite entre maladie chronique et SMét que nous avons observée dans notre étude pourrait donc indiquer que le sMét serait utile en santé publique en tant qu'indicateur clé du risque de maladie.

\section{Limites}

L'utilisation des données de l'ECMS a fait que la taille de notre échantillon n'a pas permis l'obtention d'estimations déclarables pour certaines covariables importantes et certaines caractéristiques sociodémographiques. Notre étude a été limitée à l'échelle nationale car elle ne permettait pas l'obtention d'estimations à l'échelle régionale. Par ailleurs, l'utilisation de renseignements autodéclarés pour le tabagisme ou l'activité physique durant les loisirs pourrait également constituer une limite. Étant donné l'absence de variables pertinentes pour mesurer le diabète non diagnostiqué, la portée de notre définition est limitée et l'interprétation doit se faire avec prudence. Pour limiter les effets des 
variables confusionnelles, nous avons fait des ajustements selon l'IMC, l'âge et le sexe dans les analyses multivariées. Le retrait des données manquantes pourrait avoir contribué à une erreur systématique par défaut dans le cadre de nos projections de risque de diabète, compte tenu de la tendance, pour l'IMC, à une proportion plus importante de données manquantes chez les femmes. Cependant, puisque les données manquantes chez les femmes ne représentent qu'une faible proportion des réponses pour l'IMC chez les femmes, ce retrait ne devrait pas fausser nos résultats.

\section{Conclusion}

Le SMét est une affection étroitement associée à des facteurs comme l'obésité, l'origine ethnique et l'activité physique durant les loisirs. Notre étude révèle un profil différentiel d'affection par le SMét selon certaines sous-populations et met en lumière l'existence d'une association entre le SMét et certaines maladies chroniques de premier $\operatorname{plan}^{45}$. Comme le taux de maladie chronique non diagnostiquée est significativement plus élevé chez les Canadiens atteints de SMét qu'au sein de l'ensemble de la population, et comme le taux de maladie chronique future est également plus élevé chez ces personnes, il pourrait être utile que les cliniciens considèrent, outre l'obésité, le SMét comme indicateur de maladie chronique, et que les responsables de l'élaboration des politiques en matière de santé publique tiennent compte de ce syndrome au moment du choix des activités préventives en santé des populations.

\section{Remerciements}

$\mathrm{M}^{\text {me }}$ Deepa P. Rao a reçu une bourse d'études doctorales Frederick Banting et Charles Best de la direction de l'application des connaissances de l'initiative Renouvellement des soins de santé fondés sur des données probantes des Instituts de recherche en santé du Canada (IRSC). M. D. Krewski est titulaire d'une chaire de recherche industrielle en science des risques du Conseil de recherches en sciences naturelles et en génie du Canada (CRSNG).

L'Enquête canadienne sur les mesures de la santé a été menée par Statistique
Canada en partenariat avec Santé Canada et l'Agence de la santé publique du Canada, avec un financement du gouvernement fédéral du Canada.

Nous tenons à remercier M. Michael J. Pencina de l'Université de Boston, qui nous a fourni la macro SAS pour calculer les projections de risque de MCV, de même que $M^{\text {me }}$ Laura Rosella et $M$. Michael Lebenbaum de Santé publique Ontario, qui nous a aidés à utiliser l'algorithme DPoRT pour les projections de risque de diabète.

\section{Références}

1. Morgan MW, Zamora NE, Hindmarsh MF An inconvenient truth: a sustainable healthcare system requires chronic disease prevention and management transformation. Healthc Pap. 2007;7:6-23

2. Mirolla M. The cost of chronic disease in Canada. Ottawa (Ont.) : The Chronic Disease Prevention Alliance of Canada; 2004.

3. Rapoport J, Jacobs P, Bell NR, Klarenbach S. Pour une mesure plus précise du fardeau économique associé aux maladies chroniques au Canada. Maladies chroniques au Canada. 2004;25:15-23.

4. National Cholesterol Education Program (NCEP) Expert Panel on Detection, Evaluation, and Treatment of High Blood Cholesterol in Adults (Adult Treatment Panel III). Third Report of the National Cholesterol Education Program (NCEP) Expert Panel on Detection, Evaluation, and Treatment of High Blood Cholesterol in Adults (Adult Treatment Panel III) final report. Circulation. 2002;106:3143-421.

5. Mottillo S, Filion KB, Genest J et collab. The metabolic syndrome and cardiovascular risk a systematic review and meta-analysis. J Am Coll Cardiol. 2010;56:1113-32.

6. Ford ES, Li C, Sattar N. Metabolic syndrome and incident diabetes: current state of the evidence. Diabetes Care. 2008;31: 1898-904.

7. Esposito K, Chiodini P, Colao A, Lenzi A, Giugliano D. Metabolic syndrome and risk of cancer: a systematic review and metaanalysis. Diabetes Care. 2012;35:2402-11.
8. Chen J, Muntner P, Hamm LL et collab. The metabolic syndrome and chronic kidney disease in US adults. Ann Intern Med. 2004;140:167-174.

9. Bonora E, Targher G, Formentini $G$ et collab. The metabolic syndrome is an independent predictor of cardiovascular disease in type 2 diabetic subjects. Prospective data from the Verona Diabetes Complications Study. Diabet Med. 2004;21: $52-8$.

10. Cameron AJ, Shaw JE, Zimmet PZ. The metabolic syndrome: prevalence in worldwide populations. Endocrinol Metab Clin North Am. 2004;33:351-75.

11. Magliano DJ, Cameron A, Shaw JE, Zimmet PZ. Epidemiology of metabolic syndrome. Dans : Ekoé JM, Rewers M, Williams R, Zimmet P (dir.). Epidemiology of diabetes mellitus. $2^{\text {e }}$ éd. Chichester (UK) : WileyBlackwell; 2008. p. 31-49.

12. Grundy SM. Does a diagnosis of metabolic syndrome have value in clinical practice? Am J Clin Nutr. 2006;83:1248-51.

13. Riediger ND, Clara I. Prevalence of metabolic syndrome in the Canadian adult population. CMAJ. 2011;183:E1127-34.

14. Ervin RB. Prevalence of metabolic syndrome among adults 20 years of age and over, by sex, age, race and ethnicity, and body mass index: United States, 20032006. Natl Health Stat Report. 2009;13:1-7.

15. Alberti KG, Zimmet P, Shaw J. Metabolic syndrome - a new world-wide definition. A consensus statement from the International Diabetes Federation. Diabet Med. 2006;23:469-80.

16. Alberti KG, Eckel RH, Grundy SM et collab. Harmonizing the metabolic syndrome: a joint interim statement of the International Diabetes Federation Task Force on Epidemiology and Prevention; National Heart, Lung, and Blood Institute; American Heart Association; World Heart Federation; International Atherosclerosis Society; and International Association for the Study of Obesity. Circulation. 2009;120:1640-5.

17. Grundy SM. Metabolic syndrome: a multiplex cardiovascular risk factor. J Clin Endocrinol Metab. 2007;92:399-404. 
18. Hivert MF, Grant RW, Shrader P, Meigs JB. Identifying primary care patients at risk for future diabetes and cardiovascular disease using electronic health records. BMC Health Serv Res. 2009;9:170.

19. Statistique Canada. Guide de l'utilisateur des données de l'Enquête canadienne sur les mesures de la santé (ECMS) : cycle 1. [Internet]. Ottawa (Ont.) : Statistique Canada; 2011. Consultable en ligne à la page : http://www23.statcan.gc.ca/imdb -bmdi/pub/document/5071_D2_T1_V1-fra .htm

20. Statistique Canada. Enquête canadienne sur les mesures de la santé : définitions, sources de données et méthodes - Annexe 9 : taux de réponse selon le site [Internet]. Ottawa (Ont.) : Statistique Canada; 2011. Consultable en ligne à la page : http://www23.statcan.gc.ca/ imdb-bmdi/pub/document/chms-ecms-guide/ app-ann9-fra.htm

21. Giroux S. Enquête canadienne sur les mesures de la santé : aperçu de la stratégie d'échantillonnage. Rapports sur la santé, 2007;18 Suppl:35-40.

22. Statistique Canada. Enquête canadienne sur les mesures de la santé (ECMS), Cycle 1 vague 1 : spécifications des variables dérivées (VD) [Internet]. Ottawa (Ont.) : Statistique Canada; 2010. Consultable en ligne à la page : http://www23.statcan.gc .ca/imdb-bmdi/pub/document/5071_D3_ T9_V2-fra.htm

23. Statistique Canada. Âge et sexe, âge médian selon le sexe et les ratios de groupes d'âge pour les deux sexes, pour le Canada, les provinces et les territoires [Internet]. Ottawa (Ont.) : Statistique Canada; 2010. Consultable en ligne à la page : http:// www12.statcan.gc.ca/census-recensement/ 2006/dp-pd/hlt/97-551/pages/page.cfm? Lang $=\mathrm{F} \& \mathrm{Geo}=\mathrm{PR} \&$ Code $=01 \&$ Table $=2 \&$ Data $=$ Count\&Sex $=1 \&$ StartRec $=1 \&$ Sort $=$ $2 \&$ Display $=$ Page

24. Statistique Canada. Plus haut niveau de scolarité atteint pour la population âgée de 25 à 64 ans, chiffres de 2006 pour les deux sexes, pour le Canada, les provinces et les territoires [Internet]. Ottawa (Ont.) : Statistique Canada; 2010. Consultable en ligne à la page : http://www12.statcan.gc .ca/census-recensement/2006/dp-pd/hlt/97 $-560 /$ pages $/$ page.$c f m ?$ Lang $=F \& G e o=P R \&$ Code $=01 \&$ Table $=1 \&$ Data $=$ Count $\&$ Sex $=1 \&$ StartRec $=1 \&$ Sort $=2 \&$ Display $=$ Page
25. Statistique Canada. Âge et sexe, chiffres de 2006 pour les deux sexes, pour le Canada, les provinces et les territoires [Internet]. Ottawa (Ont.) : Statistique Canada; 2010. Consultable en ligne à la page : http:// www12.statcan.gc.ca/census-recensement /2006/dp-pd/hlt/97-551/pages/page.cfm? Lang $=\mathrm{F} \& \mathrm{Geo}=\mathrm{PR} \&$ Code $=01 \&$ Table $=1 \&$ Data $=$ Count $\&$ Sex $=1 \&$ StartRec $=1 \&$ Sort $=2 \&$ Display $=$ Page

26. Statistique Canada. Portrait ethnoculturel du Canada [Internet]. Ottawa (Ont.) : Statistique Canada; 2010. Consultable en ligne à la page : http://www12.statcan.ca/ census-recensement/2006/dp-pd/hlt/97-562/ pages $/$ page. $\mathrm{cfm}$ ?Lang $=\mathrm{F} \& \mathrm{Geo}=\mathrm{PR} \&$ Code $=$ $01 \&$ Table $=1 \&$ Data $=$ Dist $\&$ StartRec $=1 \&$ Sort $=2 \&$ Display $=$ Page

27. Conference Board of Canada. Canadian income inequality: is Canada becoming more unequal? [Internet]. Ottawa (Ont.) : Conference Board of Canada; 2012 (mise à jour en 2013). Consultable en ligne à la page : http://www.conferenceboard.ca /hcp/hot-topics/caninequality.aspx

28. Campbell NR, Joffres MR, McKay DW et collab. Hypertension surveillance in Canada: minimum standards for assessing blood pressure in surveys. Can J Public Health. 2005;96:217-20.

29. Bryan S, Saint-Pierre Larose M, Campbell N, Clarke J, Tremblay MS. Mesure de la tension artérielle et de la fréquence cardiaque au repos dans l'Enquête canadienne sur les mesures de la santé, cycle 1. Rapports sur la santé. 2010;21:75-83.

30. Miller WG. Estimating glomerular filtration rate. Clin Chem Lab Med. 2009;47:1017-9.

31. SAS Enterprise Guide 4.1 [logiciel]. Cary (NC) : SAS Institute Inc.; 2006.

32. Rosella LC, Manuel DG, Burchill C, Stukel TA; PHIAT-DM team. A population-based risk algorithm for the development of diabetes: development and validation of the Diabetes Population Risk Tool (DPoRT). J Epidemiol Community Health. 2010;65:613-20.

33. D’Agostino RB Sr, Vasan RS, Pencina MJ et collab. General cardiovascular risk profile for use in primary care: the Framingham Heart Study. Circulation. 2008;117:743-53.
34. Ardern CI, Katzmarzyk PT. Geographic and demographic variation in the prevalence of metabolic syndrome in Canada. Can J Diabetes. 2007;31:34-6.

35. Tjepkema M. Obésité chez les adultes. Rapports sur la santé. 2006;17:9-26.

36. Polotsky HN, Polotsky AJ. Metabolic implications of menopause. Semin Reprod Med. 2010;28:426-34.

37. Xu WH, Ruan XN, Fu XJ et collab. Prevalence of the metabolic syndrome in Pudong New Area of Shanghai using three proposed definitions among Chinese adults. BMC Public Health. 2010;10:246.

38. Rojas R, Aguilar-Salinas CA, JimenezCorona A et collab. Metabolic syndrome in Mexican adults: results from the National Health and Nutrition Survey 2006. Salud Publica Mex. 2010;52:S11-8.

39. Weiss R, Dziura J, Burgert TS et collab. Obesity and the metabolic syndrome in children and adolescents. N Engl J Med. 2004;350:2362-74.

40. Grundy SM, Cleeman JI, Daniels SR et collab. Diagnosis and management of the metabolic syndrome - An American Heart Association/National Heart, Lung, and Blood Institute scientific statement. Circulation. 2005;112:2735-52.

41. Ford ES. The metabolic syndrome and mortality from cardiovascular disease and allcauses: findings from the National Health and Nutrition Examination Survey II Mortality Study. Atherosclerosis. 2004;173:309-14.

42. Manuel DG, Rosella LC, Tuna M, Bennett C. How many Canadians will be diagnosed with diabetes between 2007 and 2017? Assessing population risk. ICES Investigative Report. Toronto (Ont.): ICES; 2010.

43. Reaven GM. The metabolic syndrome: requiescat in pace. Clin Chem. 2005;51: 931-8.

44. Kip KE, Marroquin OC, Kelley DE et collab. Clinical importance of obesity versus the metabolic syndrome in cardiovascular risk in women: a report from the Women's Ischemia Syndrome Evaluation (WISE) study. Circulation. 2004;109:706-13.

45. Starfield B. The hidden inequity in health care. Int J Equity Health. 2011;10:15. 